\title{
Nuevas tecnologías aplicadas al ocio terapéutico: Proyecto ACTIVA
}

\author{
Rocío Zaragoza Martín \\ Departamento de Pedagogía-Producto-Mercado \\ Asociación de Investigación de la Industria del Juguete (AIJU) \\ Ibi (Alicante), España \\ rzaragoza@aiju.info \\ María Costa Ferrer \\ Departamento de Pedagogía-Producto-Mercado \\ Asociación de Investigación de la Industria del Juguete (AIJU) \\ Ibi (Alicante), España \\ Noemí Rando Hernández \\ Departamento de Pedagogía-Producto-Mercado \\ Asociación de Investigación de la Industria del Juguete (AIJU) \\ Ibi (Alicante), España \\ Marta Yáñez Fernández \\ Departamento de Pedagogía-Producto-Mercado \\ Asociación de Investigación de la Industria del Juguete (AIJU) \\ Ibi (Alicante), España
}

\begin{abstract}
The combination of games and technology opens up new possibilities for entertainment and also enables integral development of individuals by enhancing highly valued skills in a range of contexts (school, work, therapy, etc). Likewise, it provides versatility in generating contents and accessing them in a natural way, which is particularly favourable for games of all ages. From AIJu, in particular from the Department of Pedagogy (New Technologies and Recreational Therapy Area), work is carried out on recreational development activities to aid e-inclusion, as well as on improving the different capacities/skills (cognitive, physical, socio-emotional, etc.) for communities that are most excluded from the new technologies. Our team works using this media to bring the benefits of games, toys and new technologies closer to new users. An example of this is the ACTIVA research project: The development of a system of active therapy based on physical exercise that enables muscular strengthening in patients with Parkinson's through recreation and the latest Information and Communication Technologies. The main aim of the ACTIVA project is to provide the base for development of an interactive game of highly recreational and therapeutic value based on a combination of physical exercise and advanced visual and communication technologies in order to promote socialisation and entertainment and to improve motor skills and muscular dexterity for people suffering from Parkinson's disease.
\end{abstract}

Keywords: therapeutic leisure, physical and cognitive training, Parkinson's disease, e-inclusion 


\section{Resumen}

La combinación de juegos y tecnologías ofrece nuevas posibilidades de entretenimiento y permite, a su vez, el desarrollo integral de los individuos mejorando capacidades de gran valor para diferentes tipos de contextos (escolar, profesional, terapéutico, etc.). Así mismo, aporta versatilidad en la generación de contenidos y en el acceso a los mismos de forma natural, lo que favorece especialmente el juego para todas las edades. Desde AIJu, y más concretamente desde el Departamento de Pedagogía (Área de Nuevas Tecnologías y Ocio Terapéutico), trabajamos en desarrollos lúdicos que contribuyen a la e-inclusión y a la mejora de diferentes capacidades/habilidades (cognitivas, físicas, socio-afectivas, etc.) de los colectivos más alejados del uso de las nuevas tecnologías. Nuestro equipo trabaja a través de este medio para acercar los beneficios del juego, los juguetes y las nuevas tecnologías a nuevos usuarios. Ejemplo de ello es el proyecto de investigación ACTIVA: desarrollo de un sistema de terapia activa basada en el ejercicio físico que permita el fortalecimiento muscular en enfermos de Parkinson a través del ocio y las nuevas tecnologías de la información y la Comunicación. El proyecto ACTIVA tiene como principal objetivo establecer la base para el desarrollo de un juego interactivo de alto valor lúdico y terapéutico basado en la combinación del ejercicio físico con tecnologías avanzadas de visualización y comunicación para la promoción de la socialización, el entretenimiento y la mejora de las habilidades motoras y musculares en personas con Parkinson.

Palabras clave: ocio terapéutico, entrenamiento físico y cognitivo, Parkinson, e-inclusión

\section{La importancia de crear nuevas alter- nativas al ocio de calidad}

El juego ha sido abordado desde disciplinas científicas muy diversas. La sociología y la antropología (Huizinga, 1943) la psicología (Piaget, Lorenz, y Erikson) y la pedagogía (Bruner, 1988) han profundizado en el tema con el fin de explicar la influencia y los efectos del juego en la existencia humana y destacan, en la mayor parte de las investigaciones, los importantes efectos de este tipo de actividad sobre el bienestar subjetivo y objetivo de los individuos. Asimismo, reivindican la importancia de utilizar las potencialidades del ocio y del juego como una herramienta especialmente adecuada para el desarrollo de programas de intervención en diferentes contextos.

Los beneficios aportados por la actividad lúdica a la intervención aparecen, una vez más, al considerar las áreas que los expertos (Baur, y Egeler, 2001; Tabarez Fernández, 2005; y García, y Gómez, 2003) señalan como prioritarias para la mejora de la calidad de vida de este colectivo y comprobar su absoluta coincidencia con las potencialidades del juego como herramienta para la intervención:
- Promoción de la salud: a través de los juegos de ejercicio y actividades lúdicas que trabajan las habilidades cognitivas y mejoran la condición física.

- Promoción de la autonomía: a través de la mejora de las condiciones físicas, psicológicas y contextuales y de la creación de nuevas oportunidades para el aprendizaje.

- Mantenimiento del apoyo social: el entorno de juego favorece la consolidación de redes sociales, la comunicación y el contacto intergeneracional.

La actividad evita algunos factores como el aburrimiento, la rutina, el aislamiento y la soledad. Si la actividad elegida es grupal, agrega elementos de mucho interés para acrecentar una mejor calidad de vida: el grupo actúa emocionalmente de sostén, de red, aporta interlocutores que escuchan y a los que se aprende a escuchar, con pares que comparten similares condiciones o a veces estilos de vida. El grupo proporciona además la posibilidad de nuevos vínculos, tan necesarios en un proceso marcado por la soledad. 
Como se ha comentado, el juego, entre otras cosas, es capaz de generar entornos de alta motivación, lo que favorece el seguimiento de los tratamientos. Cualquier programa dirigido a la promoción de un envejecimiento saludable puede verse beneficiado por la impronta que el enfoque lúdico le aporta.

La enfermedad de Parkinson es el trastorno neurodegenerativo crónico con mayor cantidad de personas mayores afectadas, después del mal

de Alzheimer. Afecta al $1 \%$ de la población mundial de más de 60 años de edad y, de acuerdo con estimaciones de la Organización Mundial de la Salud, hay cerca de 6 millones de afectados. Se prevé que su prevalencia se duplicará para el 2030.

\section{Videoconsolas para la rehabilitación física en personas con Parkinson}

Con la enfermedad de Parkinson surgen múltiples síntomas motores como la rigidez, bloqueos, arrastre de pies al caminar, pérdida de postura, de equilibrio, de movilidad y de destreza en movimiento, entre otros. Así, uno de los objetivos del tratamiento de estos pacientes es rehabilitar, o al menos mantener, la funcionalidad del movimiento.

Se están realizando estudios desde distintos centros de innovación tecnológica y universidades que están observando los beneficios que aportan las nuevas videoconsolas interactivas que requieren movimiento físico y concentración por parte de los usuarios.

Los resultados obtenidos hasta el momento muestran buenas perspectivas; somos conscientes de que los ejercicios no pueden detener la evolución de la enfermedad, pero mejoran la fuerza corporal para que la persona se sienta menos incapacitada.

La multinacional Sony Computer Entertainment aprobó el proyecto PS3Grid sobre investigación biomédica, en colaboración con la Unidad de Investigación en Informática Biomédica del Instituto Municipal de Investigación Médica y la Universidad Pompeu Fabra de Barcelona. Se trata de un proyecto de cálculo utilizando un nuevo dispositivo PSGRId para comprender la causa de enfermedades como el Parkinson.
Por otro lado, la Universidad de Stanford, California, está realizando estudios con la consola Wii de Nintendo y su juego Wii Sports con el que se está observando una ligera progresión en la enfermedad. Se han propuesto extraer pruebas de la efectividad a corto o largo plazo de ejercicios como los que requiere Wii Sports. El videojuego se está usando como instrumento para tratar a 30 enfermos de Parkinson y su objetivo es demostrar cuál es el más útil de los ejercicios que se incluyen en el juego para los pacientes. Los primeros resultados han sido positivos: indican pequeñas mejoras en los pacientes que han alcanzado por el momento el $98 \%$ de los objetivos marcados. El doctor Hertz, profesor asistente de terapia ocupacional en la Escuela de Ciencias Aliadas de la Salud, del Medical College de Georgia, y partícipe en este proyecto afirma que el sistema Wii, al ser interactivo, propicia ciertos movimientos funcionales para poder dar respuesta, siendo un mecanismo eficaz para el trabajo con los pacientes de Parkinson.

Los estudios realizados hasta hoy en los que se han aplicado nuevas tecnologías ofrecen un panorama muy positivo de la capacidad de estas para ayudar a estos enfermos. Estas tecnologías, unidas a las actividades de ocio, nos aportan alternativas que mejoran la vida de los pacientes frenando la degeneración neuronal y proporcionándoles una mayor autonomía en sus tareas diarias.

\section{Proyecto ACTIVA: ocio y nuevas tec- nologías para la mejora física en per- sonas con Parkinson}

El proyecto, coordinado por AIJU y cofinanciado por el Ministerio de Industria, Turismo y Comercio y por el Fondo Social Europeo dentro del Plan Nacional de Investigación Científica, Desarrollo e Innovación Tecnológica 2008-2011 (Plan Avanza), surge como respuesta a una serie de necesidades actuales de la Asociación de Parkinson Madrid, extensible a todas las asociaciones de Parkinson nacionales.

Las tecnologías de la información y de la comunicación (TIC) irrumpen con fuerza en la sociedad mientras el colectivo de personas afectadas por la enfermedad de Parkinson necesitan y demandan 
nuevas formas de ayuda para enfrentarse a su vida cotidiana.

Este colectivo se enfrenta a importantes barreras que dificultan el uso y disfrute de sus ventajas y posibilidades. Considerando las concusiones obtenidas en numerosas investigaciones sobre el juego y las nuevas tecnologías, parece evidente que la combinación de ambos elementos permitiría intervenir de forma simultánea e innovadora sobre los aspectos considerados esenciales en la intervención social y de salud en la gente con Parkinson. Además, basandose en la evidencia de la influencia positiva de la actividad lúdica sobre la satisfacción vital y la calidad de vida, el equipo de investigadores de AIJU plantearon esta propuesta que permitirá explorar científicamente cómo la combinación de juego, el ejercicio físico y tecnologías avanzadas de la información y la comunicación puede ser utilizada como herramienta de ocio para la prevención y la rehabilitación de los efectos negativos asociados a la enfermedad de Parkinson (temblores, rigidez, lentitud de movimientos, etc.).

El principal objetivo de ACTIVA es establecer la base para el desarrollo de un juego interactivo de alto valor lúdico y terapéutico basado en la combinación del ejercicio físico con tecnologías avanzadas de visualización y comunicación para la promoción de la socialización, el entretenimiento y la mejora de las habilidades motoras y musculares en personas con Parkinson. Además, con los requerimientos de usabilidad específicos de este colectivo, que, basados en las tecnologías de la información y de la comunicación más avanzadas (TIC), ofrecen nuevas opciones de ocio mientras mejoran sus habilidades funcionales y sociales.

Para este fin, se utilizarán Wiimotes de la consola Wii de Nintendo como soporte de interacción de los juegos desarrollados. Además, los juegos interactivos están integrados con un sistema de información del Parkinson que permitirá a los terapeutas gestionar la rehabilitación de sus usuarios. Para ello, podrán, por un lado, seleccionar los juegos establecidos para cada usuario y los niveles de dificultad de los mismos y, por otro lado, obtener los resultados de los pacientes recogiendo información de gran utilidad sobre la evolución y el
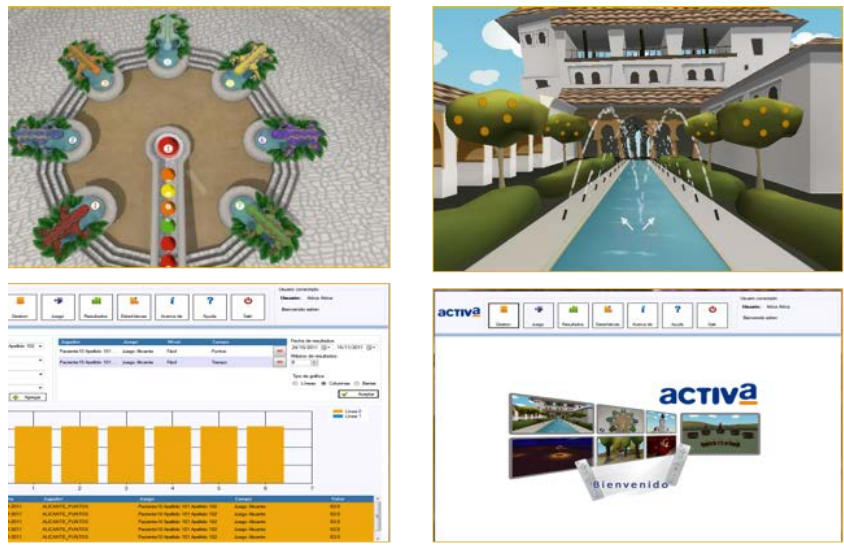

Figura 1: Ejemplos gráficos de algunos de los juegos $y$ del módulo de gestión integrados en ACTIVA
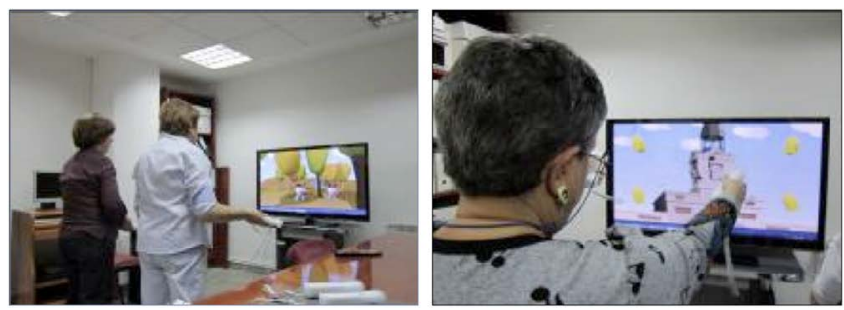

Figura 2. Usuarios participantes en la validación de aCtiva en la Asociación de Parkinson Madrid

desempeño de cada usuario en su rehabilitación a través de los juegos desarrollados.

Cada tipología de juego vendrá asociada a una capacidad física específica que se pretende mejorar. La interacción con la aplicación es una interfaz tangible e intuitiva, donde el usuario, gracias al movimiento físico del mando de la Wii, podrá dar respuesta. Además, estas interfaces permiten la estimulación sensorial de los usuarios a través de la emisión de movimientos vibratorios. El mando de la Wii incluye un sensor de movimiento de triple eje que permite detectar la señal inalámbrica a un radio de 10 metros de la consola. El mando también incluye un altavoz, una función de vibración y un puerto de expansión, y se puede utilizar como puntero en un radio de 5 metros de la pantalla. Además, está compuesto por diferentes botones destinados a acciones diferentes (apagado, encendido, etc.).

La aplicación lúdica planteada tendrá dos partes diferenciadas: 
- Área de configuración terapéutica y de resultados (personalización de sesiones y monitorización).

- Área de juego: conjunto de juegos grupales e individuales

El programa metodológico diseñado en ACTIVA parte de la prioridad de involucrar tanto a los usuarios potenciales como a los expertos en Parkinson en todas las fases del proyecto. El diseño de la investigación, basado en la triangulación metodológica, combina técnicas cualitativas y cuantitativas de recogida y análisis de datos que están siendo utilizadas de forma simultánea en función de los objetivos específicos de cada módulo de trabajo. ACTIVA ha acogido a expertos en creación de productos lúdicos de alto valor terapéutico, especialistas en realidad virtual y TIC, expertos en Parkinson y usuarios finales para la creación del primer prototipo de juego basado en las tecnologías más avanzadas de la información y la comunicación para la mejora de las capacidades físicas y funcionales de las personas que están afectadas por la enfermedad de Parkinson.

En estos momentos, el prototipo, implantado en la Asociación de Parkinson Madrid, está siendo probado por un gran número de expertos en la en- fermedad de Parkinson y por 150 pacientes para su validación en las diferentes modalidades de uso preestablecidas: juego individual, juego colectivo juego y en línea (on-line) entre centros.

Los primeros resultados que se están obteniendo en la validación de ACTIVA son muy satisfactorios y alentadores:

- Según los expertos en atención a las personas con Parkinson, la plataforma ACTIVA es un instrumento útil para la atención de las prioridades de intervención establecidas en su labor profesional cotidiana.

- Los expertos en Parkinson participantes en el Proyecto ACTIVA consideran que el manejo de la plataforma es accesible y sus contenidos motivadores.

- Las personas que padecen la enfermedad de Parkinson participantes en el Proyecto ACTIVA perciben los beneficios que para la prevención, la rehabilitación y el aprendizaje ofrece la plataforma lúdica generada por la investigación.

- Las tecnologías de realidad virtual generan entornos de alta motivación en los colectivos de personas con Parkinson participantes en el Proyecto ACTIVA.

\section{Referencias}

Baur, R. y Egeler, R. (2001). Gimnasia, juego y deporte para mayores. Barcelona: Paidotribo.

Bruner, J. S. (1988). Desarrollo cognitivo y educación. Madrid: Morata.

García, P. y Gómez, A. (2003). Efecto de los talleres de Ocio sobre el bienestar subjetivo en las personas mayores. Universidad de Málaga. Revista de Psicología Social. 18 (1). 3547.

Huizinga, J. (1943). Homo Ludens: el juego y la cultura. Alianza Editorial. ISBN 9788420635392.

Piaget, J., Lorenz, K. y Erikson, E. H. (1982). Juego y Desarrollo. Barcelona: Crítica.

Tabarez Fernández, J. (2005). El Ocio como dispositivo para la potenciación del desarrollo humano. Málaga: Ediciones Aljibe. 